\title{
Maklink Graph in Conflict-Free Airline Network Designing
}

\section{Yi-Ye Zhou, 2, a , Deng-Kai Yao ${ }^{1,2, ~ b ~}$, Qian-Rui Sun, Qi-Ke Wu W $^{1,2, c}$}

\author{
${ }^{1}$ Air Traffic Control and Navigation College, Air Force Engineering University, Xi'an, Shaanxi, \\ 710051, China \\ ${ }^{2}$ National Key Laboratory of Air Traffic Collision Prevention, Xi'an, Shaanxi, 710051, China \\ a zyyroyal@qq.com, ${ }^{b}$ yao305@163.com, ${ }^{c}$ 308541465@qq.com
}

Keywords: Maklink graph, airline network, obstacle avoidance, Dijkstra algorithm

\begin{abstract}
This paper focuses on the issue of designing of conflict-free airline network Based on the free connecting lines and undirected graph structured by Maklink Graph method, a free airspace model was established, which provided alternative air passages for restricted airspace of airline network. Take the impact of restricted airspace on airline network into consideration, a fast airspace conflict detection algorithm was proposed to realize the whole airspace conflict detection for airline network, and invoked the Dijkstra algorithm for path replanning of airlines to structure the drivable airline network with the consideration of restricted airspaces.
\end{abstract}

\section{Introduction}

Airline network is a kind of significance physical space to accomplish air transportation of both travellers and goods, and its construction has a great impact on the safety, efficiency and economy of air traffic flow. Therefore, the aviation developed countries attach great importance to airline network designing. At present the correlational research would be divided into three parts: local airline network planning [1], global airline network planning [2] and air highway designing [3]. The research above focused mostly on operating cost reduction of airline network without the restricted airspaces in consideration. In fact, existing airspace contains a mass of Prohibited Area, Restricted Area and Danger Area, PRD for short, which fragments the airspace runtime environment [4]. Figure 1 shows an example of airline network in fragmented airspace runtime environment.

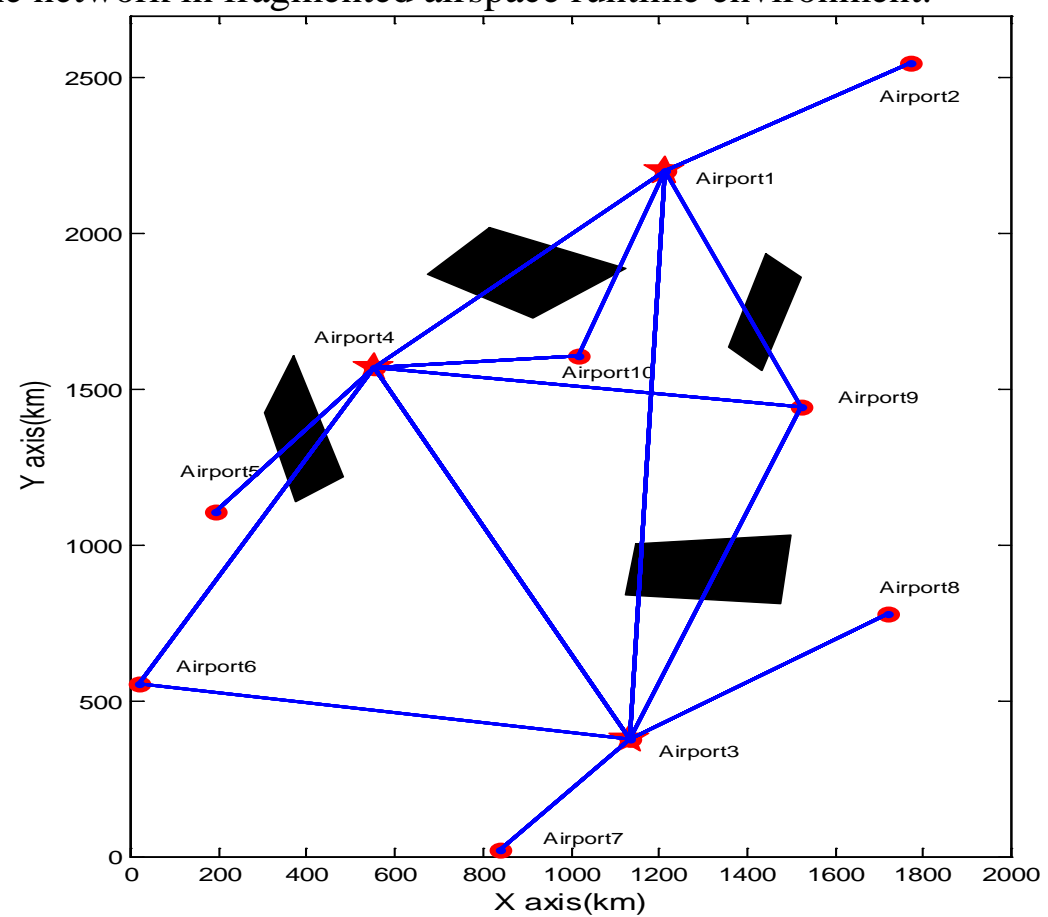

Figure 1 An example of airline network in fragmented airspace runtime environment

The black areas are PRDs, the disabled airspace for airline network design. As can be seen from Figure 1, there are 7 airlines between airport pairs in conflict with PRDs, which leaves potential 
safety hazard for airspace operating. Existing literatures show that little studies on PRD avoidance have been done, at the present Shi-Jin Wang has established a space planning model by grid method and adopted cellular automaton to solve the problem of PRD avoidance [5]. But this method lacks of applicability due to the restriction of PRD's quantity and distribution. Therefore, this paper established a free airspace model by Maklink graph to turn the PRD avoidance problem into nodes distribution problem and designed a fast airspace conflict detection algorithm to realize the whole airspace conflict detection, and the Dijkstra algorithm was invoked to replan local airlines according to the detection results.

\section{Free Airspace Model}

Before proposing Maklink, the restrict airspaces are abstracted as polygons in 2D plane first, which conform to the fundamental appearance of restrict airspaces. On this basis, restrict airspaces are expanded to guarantee the safety margin between airline network and PRD. And then, Maklink graph theory is applied to structure the undirected network graph to provide airline network the alternative air passages for conflict avoidance. The key point of Maklink graph is to find out available free connected lines and differentiate the area of free convex polygons. The detailed principle of free airspace model based on Maklink graph is as follows:

Step1: the free connected lines can't intersect with the area of restrict airspace;

Step2: the start- and end-point of free connected lines should be both the vertexes of restrict airspace, or one of the star- and end-point is the vertex and another one is on the boundary of airspace;

Step3: every free connected line can be seen as the public edge of any pair of adjacent free convex polygons, and every free convex polygon has at least two free connected lines as its side boundaries.

Draw $l$ free connected lines out according to the method above, and the middle points of them are respectively $v_{1}, v_{2}, \ldots, v_{n}$. Connect all these middle points to accomplish the complete Maklink graph, i.e. the undirected network graph. Figure 2 shows the consequence of structuring undirected network graph.

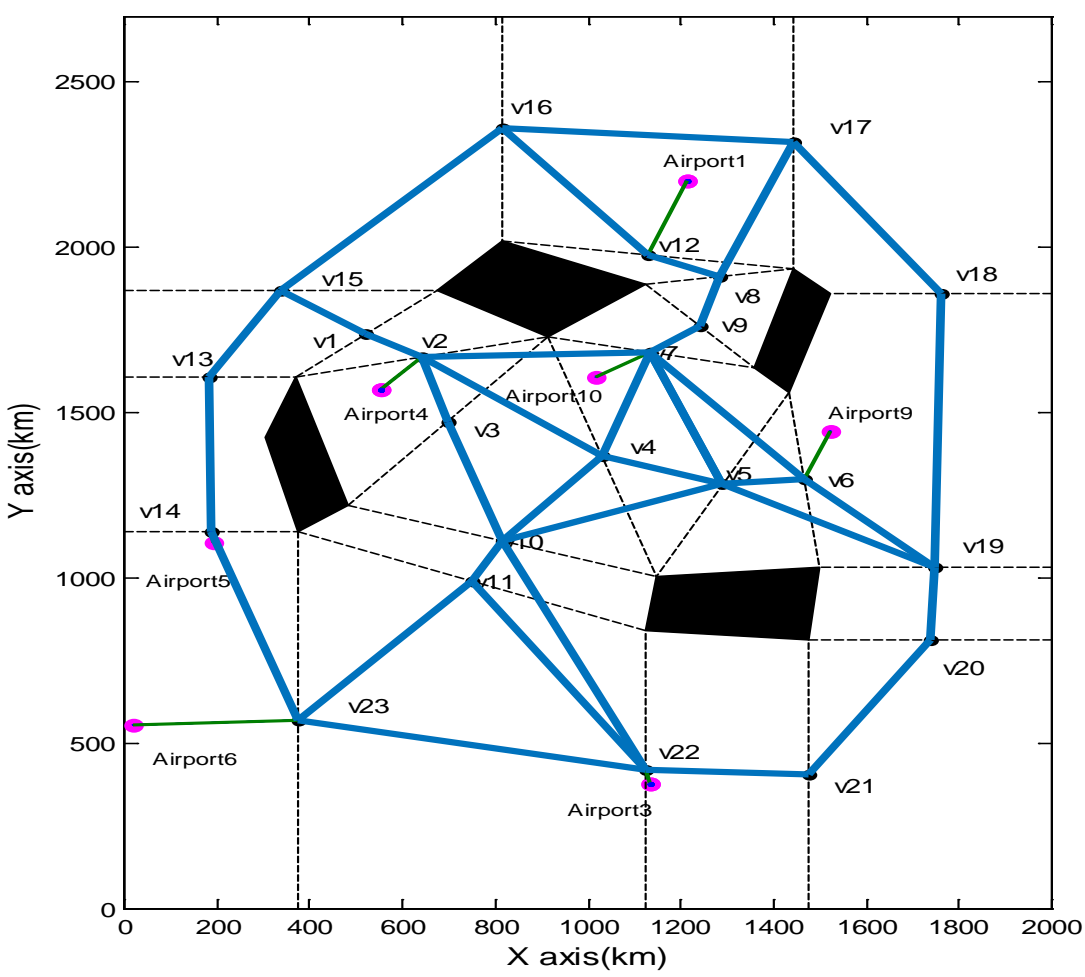

Figure 2 Consequence of structuring undirected network graph 


\section{Airspace Conflict Detection Algorithm}

To realize the whole airspace conflict detection for airline network, this paper uses Computer Graphics to precisely model each conflict in airspace, and adopts linear interpolation to segment airlines. The clockwise sampling of the free convex polygon $l$ expressed as follows:

$\left\{\begin{array}{l}\text { obstacle }{ }_{\text {p }} \text { position }(l)=\left\{P_{0}, P_{1}, \cdots, P_{i}, \cdots, P_{n}\right\} \\ P_{i}=\left(P_{i x}, P_{i y}\right) \\ P_{0}=P_{n}\end{array}\right.$

Abstract airlines as segments, and the segment $k$ is denoted as path $(k)$, while its start- and end-point respectively are $Q_{1}$ and $Q_{2}$, then this segment $k$ can be expressed in vector form:

$\left\{\begin{array}{l}Q_{t_{k}}=Q_{1}+\left(Q_{2}-Q_{t}\right) t_{k} \\ t_{k} \in[0,1]\end{array}\right.$

As we can see from equation (2), when the scaling parameter $t_{k}$ is given, the sub-line segment $\overrightarrow{Q_{1} Q_{t_{k}}}$ of segment $\overrightarrow{Q_{1} Q_{2}}$ can be confirmed. Therefore, $Q_{t_{k}}$ can be seen as a removable point on segment $\overrightarrow{Q_{1} Q_{2}}$ along with the different value of scaling parameters. In conclusion, take $\Delta t$ as step size to traverse the value of $t_{k}$ in section [0,1] , and if there is a scaling parameter $t_{k}$ which make the point $Q_{t_{k}}$ subsumed in the polygon obstacle _ position $(l)$, then it can be proved that the segment path $(k)$ is intersected with this polygon, means that there is a conflict between airline $k$ and PRD $l$. It is absolutely that smaller the step size is, the airspace conflict detection algorithm is more precise.

The time complexity of this algorithm is $O(n)$, that is to say this algorithm is efficient and requires little memory resources, which is applicative to the airspace with large number of conflicts. The detailed process of the proposed algorithm is as follows:

Input: data of the airline network which is soppose to be relan, $N_{A P}, N_{\text {path }}, \Delta t_{k}, O B S_{\text {position }}$

Output: the airlines which is conficted with PRDs

$\% \%$ conflict detection

for $i=1: N_{A P}$

\%major cycle, traverse all the airport point

store airlines in the set $N_{\text {path }}$;

for $k=1:\left|N_{\text {path }}\right| \quad \%\left|N_{\text {path }}\right|$ is number of airlines

the start- and end-point of airline $k$ are $Q_{1}(k)$ and $Q_{2}(k)$;

for $t=0: \Delta t: 1 \quad \% \Delta t$ is the step wise

$Q_{t}(k)=Q_{1}(k)+\left(Q_{2}(k)-Q_{1}(k)\right) t ; \quad \%$ structure the vector form of $N_{\text {path }}(k)$

for $l=1: L \quad \% L$ is the number of PRD

invoking function $i n(k)=$ inpolygon $\left(Q_{t}(k), O B S_{\text {position }}(l)\right)$;

if $\operatorname{in}(k) \sim=0$

$Q_{t}(k)$ is subsumed in the polygon $O B S_{\text {position }}(l)$;

store $N_{\text {path }}(k)$ in the set ConfMem ;

end if

end (for $l=1: L$ )

end (for $t=0: \Delta t: 1$ )

end (for $k=1:\left|N_{\text {path }}\right|$ )

end (for $i=1: N_{A P}$ ) 
The airline from Airport $i$ to Airport $j$ denoted by path $(i, j)$. Invoking the above algorithm, the consequence of airspace conflict detection is obstacle _ paths $=\{$ path $(1,3)$, path $(1,4)$, path $(1,9)$, path $(1,10)$, path $(3,9)$, path $(4,5)$, path $(4,6)\}$.

\section{Conflict-Free Airline Network Replanning}

The consequence of structuring undirected network graph shows that the continuous conflict-free airline network replanning problem can be translated into the shortest path problem of discrete path points, and some mature convenient algorithms, such as Dijkstra algorithm, in graph theory could be expediently invoked to the integrated airline network replanning.

According to given conditions, the complete Maklink graph can be abstracted to a weighted undirected graph $G(V, E) . V$ is a gather of $n$ vertexes including all the middle points of the free connected lines(also called path points without interference) and airport points corresponding the airlines with conflicts. $E$ is a gather of $n$ edges, and $\left[v_{i}, v_{j}\right]$ indicates an edge from $v_{i}$ to $v_{j}$ while $d_{i j}$ indicates the length of edge $\left[v_{i}, v_{j}\right]$, it's sure that $d_{i j}$ is a nonnegative weight of $\left[v_{i}, v_{j}\right]$. To facilitate the computer storage and handling, we can structure an adjacency matrix of the weighted undirected graph $G(V, E)$. Let $\operatorname{AdjMatrix}(G)$ equal to $a_{i j}$, then

$$
a_{i j}= \begin{cases}d_{i j}, & {\left[v_{i}, v_{j}\right] \neq 0} \\ \text { Inf }, & {\left[v_{i}, v_{j}\right]=0} \\ 0, & i=j\end{cases}
$$

This matrix reflects the relationship among vertex, and elements in matrix indicate the weight of edges, valuing for 0 , Inf or positive numbers. Therefore, a weight sum of a path from $v_{i}$ to $v_{j}$ (i.e. length of this path) can be expressed as

$$
D_{i j}=\sum_{k=0}^{n-1} d\left(v_{k}, v_{k+1}\right)
$$

The shortest path between any pair vertexes can be derived by equation (4) to minimize the weight sum along this path. Invoke Dijkstra algorithm to traverse all the airlines with conflicts (i.e. obstacle _ paths $=\{$ path $(1,3)$, path $(1,4)$, path $(1,9)$, path $(1,10)$, path $(3,9)$, path $(4,5)$, path $(4,6)\})$, and find out the feasible paths to resolute the conflicts between airlines and PRDs. Table 1 shows the consequence of feasible airline network replanning based on Dijkstra algorithm.

Table 1 Consequence of feasible airline network replanning

\begin{tabular}{cc}
\hline Airline & Feasible Path \\
\hline path $(1,3)$ & Airport $\rightarrow v_{12} \rightarrow v_{8} \rightarrow v_{9} \rightarrow v_{7} \rightarrow v_{4} \rightarrow v_{10} \rightarrow v_{22} \rightarrow$ Airport3 \\
\hline path $(1,4)$ & Airport $1 \rightarrow v_{12} \rightarrow v_{8} \rightarrow v_{9} \rightarrow v_{7} \rightarrow v_{2} \rightarrow$ Airport4 \\
\hline path $(1,9)$ & Airport $\rightarrow v_{12} \rightarrow v_{8} \rightarrow v_{9} \rightarrow v_{7} \rightarrow v_{6} \rightarrow$ Airport9 \\
\hline path $(1,10)$ & Airport $\rightarrow v_{12} \rightarrow v_{8} \rightarrow v_{9} \rightarrow v_{7} \rightarrow$ Airport10 \\
\hline path $(3,9)$ & Airport3 $\rightarrow v_{22} \rightarrow v_{10} \rightarrow v_{5} \rightarrow v_{6} \rightarrow$ Airport9 \\
\hline path(4,5) & Airport $4 \rightarrow v_{2} \rightarrow v_{1} \rightarrow v_{15} \rightarrow v_{13} \rightarrow v_{14} \rightarrow$ Airport5 \\
\hline path(4,6) & Airport4 $\rightarrow v_{2} \rightarrow v_{3} \rightarrow v_{10} \rightarrow v_{11} \rightarrow v_{23} \rightarrow$ Airport6
\end{tabular}

Point $v_{i}$ indicates the path point without interference, also the middle point of corresponding free connected line. According to the consequence of feasible airline network replanning in table 1, draw out the figure of conflict-free airline network showed in figure 3. 


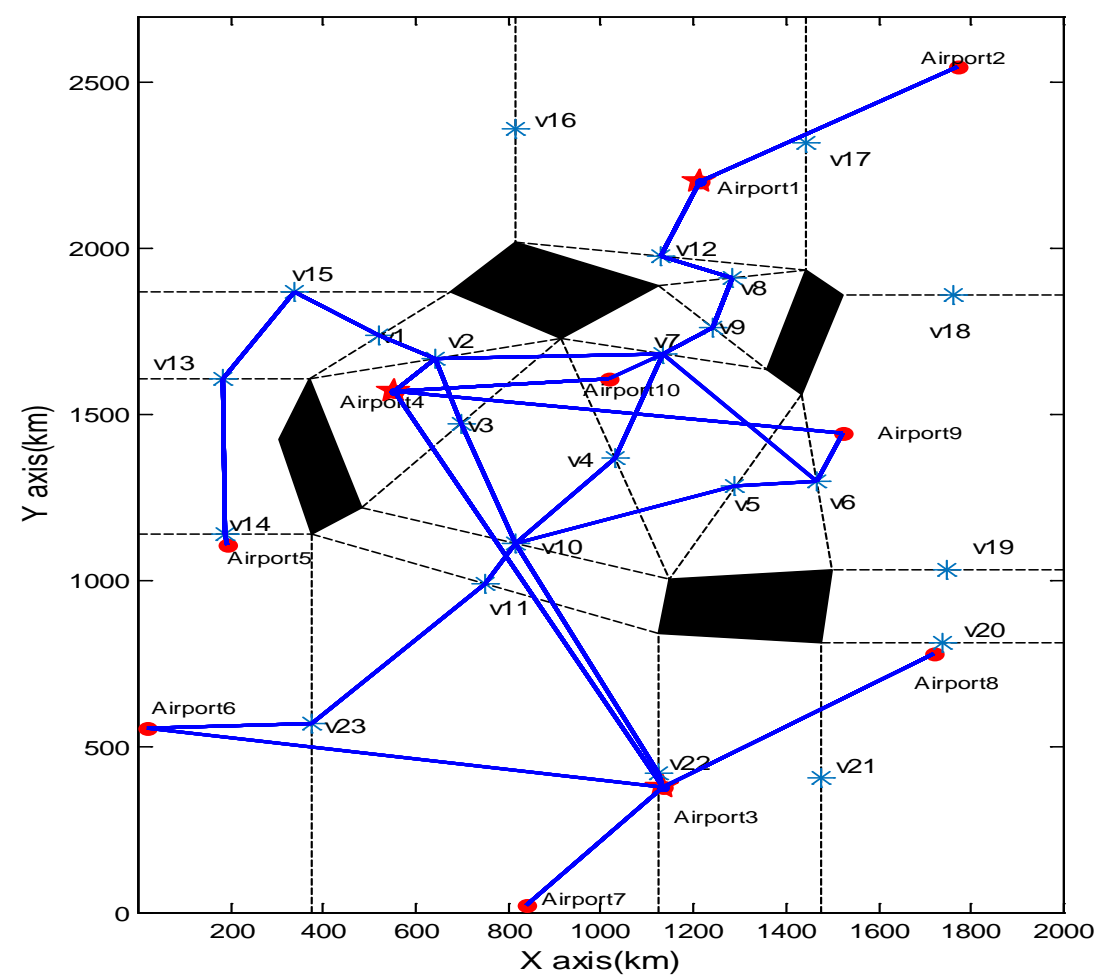

Figure 3 Conflict-free airline network

\section{Summary}

This paper considered the possible influence of PRDs in airspace runtime environment.First, based on the theory of Maklink graph, a free airspace model was established for the convenience of mathmatical description and providing alternative air passages for restricted airspace of airline network. Second, A fast airspace conflict detection algorithm was proposed for the preparation of airline network replanning,. And then, we analyzed the topological structure of the complete Maklink graph to translate the continuous conflict-free airline network replanning problem into the shortest path problem of discrete path points. In the end, Dijkstra algorithm was invoked to replan every airlines with conflict to accomplish the conflict-free airline network design. The instance analysis demonstrates that the proposed method can effectively solve the problem.

\section{References}

[1] Shuang-Zhao. Research on National Air Route Backbone Network. Beijing: Beijing University of Aeronautics and Astronautics, 2008.

[2] Zheng-Wei Xin. Research on Technique of Air Route Network. Nanjing: Nanjing University of Aeronautics and Astronautics, 2013.

[3] Arash Y, Jerome L,and John T. NextGen Flow Corridors Initial Design,Procedures,and Display Functionalities//Proceedings of the 29th Digital Avionics Systems Conference,2010: 11-19.

[4] Optimization and Evalution of Air Route Network under PRD Avoiding. Aeronautical Computing Technique, 2015, 45(4): 30-32.

[5] Shi-Jin Wang, Yan-Hui Gong and Qing-Yun Li. Research Overview on Technique of Airline Network Planning. Traffic Information and Security, 2014, 32(6): 8-10. 